\title{
Nuevos puntos de vista sobre el ataque de sulfatos y cloruros alcalinos al hormigón
}

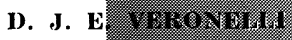

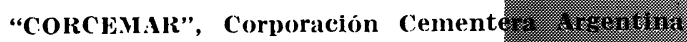

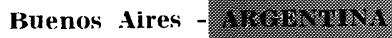

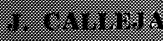

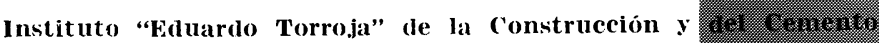

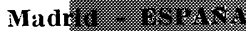

\section{R E S U M E N}

Los autores recopilan información privada, intercambiada entre sí tiempo atrás, y aportan nuevos puntos de vista sobre la posibilidad de reacciones árido-álcal:s en hormigones con áridos reactivos, producidas por compuestos alcalinos — sulfatos y/o cloruros- ajenos al cemento y procedentes del medio externo en que se encuentren los hormigones.

Emiten sus hipótesis sobre la base de recientes resultados experimentales de otros autores y señalan, de acuerdo con éstos, la necesidad de estudios más detallados, prec.sando algunos de los puntos del tema que convendría poner en claro mediante la oportuna experimentación

\section{INTRODUCCION}

Cualquier ataque químico al cemミnto y al hormigón presenta, casi siempre, características muy complejas que hacen posible, a veces, interpretaciones y explicaciones varias y distintas. Estas pueden ser mutuamente excluyentes e incluso contradictorias, o, por el contrario, complementarias y/o simultáneas.

Las acciones agresivas de los sulfatos y de los cloruros sobre el cemento hidratado dependen, entre otras circunstancias, de los cationes constituyentes de dichas sales. Cuando el catión es el calcio, tales acciones parecen más simples y tienen un carácter más específico. Cuando, por el contrario, los cationes son alcalinos, puede surgir una mayor complicación dado que a la acción de los sulfatos y de los cloruros como tales puede sumarse la de los álcalis cáusticos que eventualmente puedan formarse en el curso del ataque, como consecuencia de las reacciones que tienen lugar entre los sulfatos y los cloruros alcalinos y los componentes de la pasta hidratada del cemento. Estos álcalis pueden actuar, no solamente sobre la pasta cementicia, sino también sobre determinados áridos susceptibles a ellos. Se estaría así en el caso más complejo de un ataque mixto y simultáneo, tanto sobre el cemento (producido principalmente por los aniones) como sobre el árido reactivo (provocado mayormente por la fuerte alcalinidad de las bases formadas por los cationes). 
Estos aspectos han llamado recientemente la atención, habiendo sido objeto de consideraciones que han dado lugar a interpretaciones, las cuales se prestan a un análisis e incluso a una ampliación. Tal es el objeto de este trabajo.

\section{POSIBLES ESQUEMAS INTERPRETATIVOS}

Para D. J. E. VERONELLI (1) la acción de los sulfatos y de los cloruros alcalinos sobre los productos de hidratación del cemento portland, y más concretamente sobre la portlandita y/o los aluminatos, puede dar lugar a la formación de hidróxidos alcalinos, según los esquemas:

2)

$$
\begin{aligned}
& \mathrm{Na}_{2} \mathrm{SO}_{4}+\mathrm{Ca}(\mathrm{OH})_{2}+2 \mathrm{H}_{2} \mathrm{O} \stackrel{\text { aq. }}{\longrightarrow} \mathrm{CaSO}_{4} \cdot 2 \mathrm{H}_{2} \mathrm{O}+2 \mathrm{NaOH} \\
& 2\left[3 \mathrm{CaO} . \mathrm{Al}_{2} \mathrm{O}_{3} \cdot 6 \mathrm{H}_{2} \mathrm{O}\right]+3 \mathrm{Na}_{2} \mathrm{SO}_{4}+25 \mathrm{H}_{2} \mathrm{O} \rightarrow \\
& \rightarrow 3 \mathrm{CaO} \mathrm{Al}_{2} \mathrm{O}_{3} \cdot 3 \mathrm{CaSO}_{4} \cdot 31 \mathrm{H}_{2} \mathrm{O}+2 \mathrm{Al}(\mathrm{OH})_{3}+6 \mathrm{NaOH} \\
& 2 \mathrm{NaCl}+\mathrm{Ca}(\mathrm{OH})_{2}+3 \mathrm{CaO} \cdot \mathrm{Al}_{2} \mathrm{O}_{3} \cdot 6 \mathrm{H}_{2} \mathrm{O}+4 \mathrm{H}_{2} \mathrm{O} \rightarrow \\
& \rightarrow 3 \mathrm{CaO} \cdot \mathrm{Al}_{2} \mathrm{O}_{3} \cdot \mathrm{CaCl}_{2} \cdot 10 \mathrm{H}_{2} \mathrm{O}+2 \mathrm{NaOH}
\end{aligned}
$$

Es de observar que, según el esquema 2), no interviene la portlandita, y además de hidróxido sódico se formaría alúmina hidratada (gibbsita).

Para J. CALLEJA (2) el equilibrio de solubilidad y disociación del hidróxido cálcico:

$$
\mathrm{Ca}(\mathrm{OH})_{2} \Longleftrightarrow \mathrm{Ca}^{2+}+2\left(\mathrm{OH}^{-}\right)
$$

y el del sulfato cálcico:

$$
\mathrm{CaSO}_{4} \Longleftrightarrow \mathrm{Ca}^{2+}+\mathrm{SO}_{4}{ }^{2-}
$$

se acoplan en uno solo:

$$
\mathrm{Ca}(\mathrm{OH})_{2}+\mathrm{SO}_{4}{ }^{2-} \leftarrow \mathrm{CaSO}_{4}+2\left(\mathrm{OH}^{-}\right)
$$

el cual se daría en la fase líquida de la pasta de un cemento exento de álcalis y de sulfatos alcalinos solubles.

A este mismo equilibrio, si bien establecido en otras condiciones, se llegaría a través del proceso 1), ya que representado éste en forma iónica:

7) $2 \mathrm{Na}^{+}+\mathrm{SO}_{4}{ }^{2-}+\mathrm{Ca}^{2+}+2\left(\mathrm{OH}^{-}\right)+$aq. $\rightarrow \mathrm{Ca}^{2+}+\mathrm{SO}_{4}{ }^{2-}+2 \mathrm{Na}^{+}+2\left(\mathrm{OH}^{-}\right)+$aq.

mostraría que no podría haber interacciones en disolución, de no formarse - como es el caso - compuestos de escasa solubilidad y bajo grado de disociación — como son la portlandita y el yeso-, junto con compuestos muy solubles que son electrolitos fuertes disociados de forma prácticamente total —como el sulfato sódico y el hidróxido sódico-. En definitiva, a través de:

$$
\mathrm{Na}_{2} \mathrm{SO}_{4}+\mathrm{Ca}(\mathrm{OH})_{2} \longleftrightarrow \mathrm{CaSO}_{4}+2 \mathrm{NaOH}
$$

se vuelve a parar al equilibrio 6). 
En dicho equilibrio se verifica que:

9)

$\left(\mathrm{SO}_{4}{ }^{2-}\right) /\left(\mathrm{OH}^{-}\right)^{2}=\mathrm{K}$

siendo inalterable para cada temperatura y en unas condiciones dadas, pues una vez establecido, no podría haber interacciones entre el sulfato cálcico y el hidróxido cálcico, en una disolución común saturada de ambos, y en unas condiciones fijadas para dicho equilibrio.

Sin embargo, el equilibrio 6) se puede desplazar en un sentido u otro, según aumenten o disminuyan en una u otra proporción, bien sea la concentración de iones $\mathrm{SO}_{4}{ }^{2-}$ por la disolución y la disociación de los sulfatos alcalinos, fácil y rápidamente solubles y normalmente presentes en el clínker y en el cemento, o bien por la disolución y disociación de los óxidos alcalinos incluidos en los silicatos y en los aluminatos cálcicos del clínker $\left(\mathrm{KC}_{23} \mathrm{~S}_{12}\right.$ y $\mathrm{NC}_{8} \mathrm{~A}_{3}$ ).

El equilibrio 6) también se puede desplazar por la introducción o penetración de un sulfato alcalino desde el exterior en la fase líquida de los poros de la pasta de cemento.

Por lo tanto, el desplazamiento del equilibrio en un sentido u otro dependerá de las variaciones de concentración de los aniones $\mathrm{SO}_{4}{ }^{2-}$ y $\mathrm{OH}^{-}$en cada caso, con respecto a las concentraciones de equilibrio en unas condiciones dadas.

En cualquier circunstancia, todo aumento de concentración de aniones sulfato en la fase líquida de la pasta de cemento - y este es el caso de medios acuosos con sulfatos alcalinos, tanto si provienen del clínker de cemento como del medio exterior-, debe llevar aparejado un aumento proporcional de la concentración de iones hidroxilo en la misma, por solubilización y disociación de la portlandita y formación e insolubilización de yeso, para mantener el equilibrio 6), según 9). En definitiva, ello implica un consumo de portlandita como fuente aportadora de iones $\mathrm{OH}^{-}$.

Asimismo CALLEJA (2) (3) supone que en la reacción 2) de los aluminatos hidratados con los sulfatos alcalinos, bien en la forma expuesta o bien en la alternativa:

$10)^{*} \quad 6 \mathrm{NS}+3 \mathrm{C}_{4} \mathrm{AH}_{1: 3191}+32(14) \mathrm{H} \rightarrow 2\left[\mathrm{C}_{3} \mathrm{~A}(\mathrm{CS})_{33} \mathrm{H}_{31}\right]+\mathrm{AH}_{3}+6 \mathrm{NH}$

con liberación de alúmina (gibbsita) —como en 2)—, o en las alternativas:

$$
\begin{aligned}
& 3 \mathrm{~N} \overline{\mathrm{S}}+3 \mathrm{CH}+\mathrm{C}_{3} \mathrm{AH}_{6}+25 \mathrm{H} \rightarrow \mathrm{C}_{3} \mathrm{~A}(\mathrm{C} \overline{\mathrm{S}})_{3} \mathrm{H}_{31}+3 \mathrm{NH} \\
& 3 \mathrm{NS}+2 \mathrm{CH}+\mathrm{C}_{4} \mathrm{AH}_{19}+13 \mathrm{H} \rightarrow \mathrm{C}_{3} \mathrm{~A}(\mathrm{C} \overline{\mathrm{S}})_{3} \mathrm{H}_{31}+3 \mathrm{NH}
\end{aligned}
$$

con consumición de cal (portlandita) - como en 3)-, se producen cantidades de ettringita y de "álcali cáustico" que son mayores en 11) y 12) que en 10), en relación con la cantidad de aluminato o de alúmina puestos en juego en cada caso. El consumo de cal -portlandita- en 12) sustraería a parte de ésta del proceso 1), pero daría lugar, proporcionalmente, a una mayor formación de álcali cáustico".

\footnotetext{
* En este y en los siguientes esquemas:

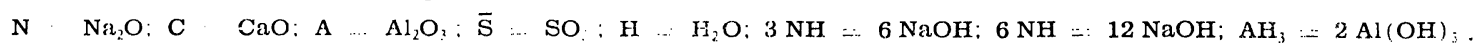


VERONELLI (1) ha sugerido -y CALLEJA (2) comparte la sugerencia- que la alcalinidad cáustica (fuerte) creada por alguno de los mecanismos señalados puede actuar sobre los áridos silícicos reactivos de los hormigones que los contengan, dando lugar a la reacción árido-álcali, en su modalidad álcali-silícica. Sobre todo en el caso de que tales hormigones sean permeables en tal medida que permitan el fácil acceso de posibles sulfatos alcalinos existentes en un medio acuoso, como puede ser el marino.

Naturalmente que los efectos de esta posible reacción árido-álcalis se sumarían, tanto a los producidos por una formación y cristalización expansiva de yeso en condiciones propicias según 1), como a los causados por la iormación de ettringita según 2), 10,11) ó 12).

Hace observar VERONELLI (1) que en el caso de ser esto así la reacción árido-álcali no se produce sólo o principalmente por los álcalis del cemento, por lo cual tampoco se puede controlar por el contenido de los mismos ni evitarla limitando drásticamente dicho contenido en el conglomerante.

Es decir, que con cementos que idealmente contuvieran $0 \%$ de álcalis podría tener lugar el deterioro de un hormigón con ár:dos reactivos en un medio acuoso salino de sulfatos y/o de cloruros alcalinos - por ejemplo, agua de mar-, por la reacción expansiva árido-álcalis, aparte de otros posibles deterioros por otras causas y mecanismos.

Esta observación abona una vez más las ideas sustentadas por CALLEJA (4), en el sentido de la discutibilidad del valor real y práctico de las limitaciones del contenido máximo admisible de álcalis $-\mathrm{Na}_{2} \mathrm{O}$ equivalente- de los cementos en las normas.

Otra observación de VERONELLI (1), consecuencia de la anterior, es que para hormigones que hayan de estar en contacto con medios acuosos que contengan sulfatos y/o cloruros alcalinos, se deben emplear áridos no reactivos (cualquiera que sea el cemento utilizado), y/o usar cementos puzolánicos aptos para inhibir la reacción árido-álcalis.

A este último respecto señala CALLEJA (2) que el empleo de cementos puzolánicos en tales condiciones sería recomendable por varios motivos. En primer lugar, por la conocida acción de las puzolanas finamente molidas, en cuanto al mecanismo de la reacción áridoálcalis, al sustituir, como consecuencia de su reactividad y gran superficie específica, unas pocas acciones localizadas, poco extensas pero muy intensas y con grandes efectos destructivos para el hormigón, por muchas acciones dispersas, muy extendidas pero poco intensas, inocuas o mucho menos nocivas para aquél. Y, en segundo lugar, por la acción química puzolánica, fijadora de la cal $-\mathrm{Ca}(\mathrm{OH})_{\check{z}}$, la cual es, a fin de cuentas y como queda expuesto, la fuente (al menos la fuente más importante) de la alcalinidad fuerte -iones $\mathrm{OH}^{-}$-, por acción de los sulfatos alcalinos sobre la pasta hidratada del cemento, según 8) y 9).

También recientemente K. PETTIFER y P. J. NIXON (5) han considerado los efectos conjuntos del ataque por sulfatos y de la reacción árido-álcalis en el hormigón con árido reactivo, producidos por sulfatos alcalinos.

Los autores describen casos prácticos en los que simultáneamente se ha observado por microscopía óptica, por análisis térmico diferencial, por difracción de rayos $\mathrm{X}$ y, en algún caso, por análisis con microsonda electrónica, la presencia de geles silícicos isotrópicos, indicativos de una reacción árido-álcalis, de cantidades moderadas o muy grandes de ettringita, índice de un fuerte ataque de sulfatos y, a veces, de portlandita secundaria. En alguno de estos casos el hormigón estaba hecho con cemento portland de bajo contenido de álcalis. En otros casos, o bien el árido era de origen marítimo y sin lavar, o el hormigón 
se amasó con agua de mar, o estuvo en contacto con materiales aportadores de sulfatos alcalinos y de calcio. En todos estos casos hubo, pues, aportación externa de compuestos salinos alcalinos (sulfatos y/o cloruros).

Los autores citados (5) tratan de explicar sus observaciones a base de la hipótesis de $\mathrm{S}$. DIAMOND (6), de que el ataque árido-álcali se produce por la acción de un hidróxido alcalino, cuya presencia en la fase líquida de la pasta de cemento, como consecuencia de una reacción de los aluminatos hidratados con los iones $\mathrm{SO}_{4}{ }^{2-}$ de los sulfatos solubles del clínker para formar ettringita, produce un aumento de la concentración de iones $\mathrm{OH}^{-}$en el líquido de los poros de la pasta.

El esquema propuesto:

$$
\begin{aligned}
& \mathrm{Ca}(\mathrm{OH})_{2} \longleftrightarrow \mathrm{Ca}^{2+}+2\left(\mathrm{OH}^{-}\right) \\
& 4 \mathrm{CaO} . \mathrm{Al}_{2} \mathrm{O}_{3} \cdot 19 \mathrm{H}_{2} \mathrm{O}+3 \mathrm{Ca}^{2+}+3 \mathrm{SO}_{4}{ }^{2-}+13 \mathrm{H}_{2} \mathrm{O} \rightarrow
\end{aligned}
$$

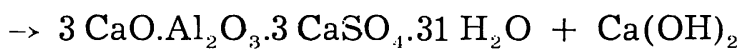

es decir:

$$
\mathrm{C}_{4} \mathrm{AH}_{19}+3 \mathrm{CS}+13 \mathrm{H} \rightarrow \mathrm{C}_{3} \mathrm{~A}(\overline{\mathrm{CS}})_{3} \mathrm{H}_{31}+\mathrm{CH}
$$

supone la formación de portlandita secundaria (no procedente de la hidrólisis de los silicatos), simultánea con la de ettringita, así como el mantenimiento de una disolución saturada de hidróxido cálcico en equilibrio. Los autores (5) admiten la posibilidad de acciones análogas por sulfatos alcalinos externos, de acuerdo con los esquemas inmediatamente precedentes 13) y 14) ó 14-I).

Sin embargo, los esquemas de los procesos 4) y 5), reunidos en 6), permiten apreciar me. jor, en virtud de 9), el hecho de que el aumento de concentración del ion sulfato por la di. solución de un sulfato alcalino eleve la concentración de iones hidroxilo hasta valores correspondientes a los de la presencia de un hidróxido alcalıno en la fase líquida. Esto permite visualizar mediante el esquema 8), la acción más directa de los sulfatos alcalinos, tanto del clínker como del exterior, así como la formación real de un hidróxido alcalino totalmente disociado, es decir, la formación de una fuerte alcalinidad cáustica en la disolución, con alta concentración de iones $\mathrm{OH}^{*}$.

En cuanto a la formación de ettringita por acción de los sulfatos sobre los aluminatos hidratados de distinta basicidad, esquemas como el 11) y el 12) muestran la necesidad de cal, mientras que esquemas como el 2) o el 10) no muestran tal necesidad. El esquema 14), en cambio, no sólo no la muestra, sino que indica la formac:ón de una portlandita secundaria.

PETTIFER y NIXON (5) presentan la imagen por microscopía electrónica y los análisis cuantitativos de azufre, aluminio y calcio por microsonda electrónica, en tres puntos de un hormigón afectado por ataque de sulfatos y por reacción árido-álcalis. Al comparar los resultados hallados por los citados autores con los valores teóricos del contenido de dichos elementos en la composición de la ettringita $(7,7 \%$ para S, $4,3 \%$ para Al y $19,3 \%$ para $\mathrm{Ca}$ ), se pueden observar, en el promedio de los tres análisis, contenidos de $\mathrm{Al}-\mathrm{y}$ por lo tanto de alúmina-, y de $\mathrm{Ca}-\mathrm{y}$ por lo tanto de cal-, muy superiores a los teóricos, así como un contenido de $\mathrm{S}-\mathrm{y}$ por lo tanto de $\mathrm{SO}_{3}$-, algo inferior (si bien en uno de los tres casos el valor hallado es prácticamente igual al teórico). Los autores señalan también que la morfología de la ettringita observada es poco usual, es decir, atípica. 
Si se admite que los valores de $\mathrm{S}\left(\mathrm{SO}_{3}\right)$ son aceptablemente próximos al teórico correspondiente a la ettringita, y que los de $\mathrm{Al}\left(\mathrm{Al}_{2} \mathrm{O}_{3}\right)$ y $\mathrm{Ca}(\mathrm{CaO})$ son más elevados, cabe pensar que junto al aluminio (alúmina) y al calcio (cal) de la ettringita la microsonda detecta y acusa los de otros compuestos que se forman estrechamente ligados con ella, al mismo tiempo y en el mismo proceso, o en procesos distintos, simultáneos o sucesivos. Sobre todo, si el radio de acción de la microsonda es mayor que el espacio ocupado por el compuesto observado —n este caso la ettringita-, o si dicho compuesto y los adyacentes son lo suficientemente microcristalinos o sub-microcristalinos, o sus agregados lo suficientemente pequeños, y si están lo bastante imbricados e interpuestos entre sí como para que se dé una circunstancia análoga, con los mismos resultados.

La mayor cantidad de aluminio (alúmina) detectada se podría explicar así por un proceso del tipo 2) ó 10), con formación simultánea de ettringita y gibbsita, mientras que la mayor cantidad de calcio (cal) podría responder a un esquema del tipo 14) ó 14-I), con formación simultánea de ettringita y portlandita secundaria.

\section{CONSIDERACIONES ADICIONALES}

La formación de alcalinidad cáustica fuerte (hidróxido alcalino), responsable de posibles reacciones árido-álcalis por la acción de sulfatos y/o de cloruros alcalinos externos (agua de mar, por ejemplo) sobre la pasta hidratada del cemento portland podría responder, en cuanto a forma, a alguno de los esquemas 1), 2), 3), 10), 11) y 12), o a un conjunto o combinación de ellos.

La formación de alúmina (gibbsita) junto con ettringita y geles isotrópicos producidos por una reacción árido-álcalis entre áridos reactivos e hidróxidos alcalinos originados por sulfatos alcalinos podría responder, en cuanto a forma, a los esquemas 2) o 10).

La formación de cal (portlandita secundaria) junto con la ettringita y los geles isotrópicos mencionados podría responder, en cuanto a forma, al esquema 14-I), atribuible a una acción más específica de sulfato cálcico (yeso).

La formación compleja, simultánea o sucesiva, de hidróxidos alcalinos, ettringita, portlandita secundaria y gibbsita (y la correlativa de geles isotrópicos) con áridos reactivos en el hormigón, podría tener lugar, en cuanto a forma, por una conjunción de los esquemas 10), 12) y 14-I), cuya "suma" — no ponderada - podría representarse así :

$$
\begin{gathered}
9 \mathrm{~N} \overline{\mathrm{S}}+3 \mathrm{CS}+2 \mathrm{CH}+5 \mathrm{C}_{4} \mathrm{AH}_{13(19)}+70(40) \mathrm{H} \rightarrow \\
\rightarrow 4\left[\mathrm{C}_{3} \mathrm{~A}(\overline{\mathrm{CS}})_{3} \mathrm{H}_{31}\right]+\mathrm{AH}_{3}+\mathrm{CH}+9 \mathrm{NH}
\end{gathered}
$$

es decir:

$$
\begin{aligned}
9 \mathrm{~N} \overline{\mathrm{S}}+ & 3 \mathrm{CS}+\mathrm{CH}+5 \mathrm{C}_{4} \mathrm{AH}_{13(19)}+70(40) \mathrm{H} \rightarrow \\
& \rightarrow 4\left[\mathrm{C}_{3} \mathrm{~A}(\mathrm{CS})_{3} \mathrm{H}_{31}\right]+\mathrm{AH}_{3}+9 \mathrm{NH}
\end{aligned}
$$

En el esquema 12) se consume más cal (portlandita) de la que se produce por vía secundaria en el esquema 14-I), lo cual se refleja también en los esquemas 15) y 15-I). Esto quie- 
re decir que para la viabilidad del proceso 12) o para la realización simultánea de los procesos 12 y 14-I) y para la formación de portlandita secundaria según 14-I), es precisa la participación de una portlandita que hay que suponer primaria - procedente de la hidrólisis de los silicatos cálcicos- en el proceso 12). De no formarse portlandita secundaria -es decir, de no darse el proceso 14-I), los procesos 10) y 12) darían lugar formalmente al 16)—:

16) $9 \mathrm{NS}+2 \mathrm{CH}+4 \mathrm{C}_{4} \mathrm{AH}_{13(19)}+51(27) \mathrm{H} \rightarrow 3\left[\mathrm{C}_{3} \mathrm{~A}(\overline{\mathrm{CS}}) \mathrm{H}_{31}\right]+\mathrm{AH}_{3}+9 \mathrm{NH}$

el cual requiere también la contribución de portlandita primaria.

De no formarse alúmina -es decir, de no darse el proceso 10-, los procesos 12) y 14) ó 14-I) darían lugar formalmente a los procesos 17) ó 17-I), en cada caso:

17) $3 \mathrm{NS}+3 \mathrm{CS}+2 \mathrm{CH}+2 \mathrm{C}_{4} \mathrm{AH}_{13(19)}+38(26) \mathrm{H} \rightarrow 2\left[\mathrm{C}_{3} \mathrm{~A}(\mathrm{C} \overline{\mathrm{S}})_{3} \mathrm{H}_{31}\right]+\mathrm{CH}+3 \mathrm{NH}$

17-I) $3 \mathrm{~N} \overline{\mathrm{S}}+3 \mathrm{CS}+\mathrm{CH}+2 \mathrm{C}_{4} \mathrm{AH}_{1.8(19)}+38(26) \mathrm{H} \rightarrow 2\left[\mathrm{C}_{3} \mathrm{~A}(\overline{\mathrm{CS}})_{3} \mathrm{H}_{31}\right]+3 \mathrm{NH}$

También el esquema 17) consume más cal de la que se produce por vía secundaria en el esquema 14), lo que indica que para su viabilidad es precisa la participación de la portlandita primaria - como en el esquema 12)—.

De no darse el proceso 12) que requiere la participación de portlandita primaria, los procesos 10) y 14) darían lugar formalmente al 18):

18) $6 \mathrm{NS}+3 \mathrm{C} \overline{\mathrm{S}}+4 \mathrm{C}_{4} \mathrm{AH}_{1.3(19)}+51(27) \mathrm{H} \rightarrow 3\left[\mathrm{C}_{3} \mathrm{~A}(\mathrm{C} \overline{\mathrm{S}})_{3} \mathrm{H}_{31}\right]+\mathrm{AH}_{3}+\mathrm{CH}+6 \mathrm{NH}$

en el cual -como en el 14) - se produce portlandita secundaria, además de gibbsita -como en el 10)-, sin participación de portlandita primaria. Este esquema responde en realidad al propuesto por PETTIFER y NIXON (5).

En resumen, el esquema 12), muestra la acción directa de los sulfatos alcalinos externos sobre los aluminatos hidratados, en presencia de cal (portlandita primaria), para dar ettringita e hidróxido alcalino.

El esquema 14) o el 14-I) por sí solos explicarían la formación de portlandita secundaria, pero no claramente la de un hidróxido alcalino y, por lo tanto, tampoco la posibilidad clara de una reacción árido-álcalis. Representan más bien de modo exclusivo un ataque por sulfato cálcico, con formación de ettringita y portlandita. Por lo tanto, es preciso admitir que, en caso de tener lugar, habría de ir acompañado de algún otro en el que se vea más clara la acción de los sulfatos alcalinos y la formación de hidróxidos alcalinos, como es el caso del esquema 12).

Los esquemas 15) y 15-I) y 18) implican una acción conjunta de sulfato alcalino y sulfato calcico, con intervención de cal (portlandita primaria) salvo en el 18), y con formación de hidróxido alcalino.

Por otra parte, los esquemas 15) y 15-I), 16) y 18) implican la formación de gibbsita y de hidróxido alcalino, y de ellos el 16) sin intervención de sulfato cálcico, pero con intervención de portlandita primaria. 
Los esquemas 1), 6) y 8) indican el equilibrio entre el sulfato y el h:dróxido cálcico en disolución saturada de ambos, regido por 9), por el cual un sulfato alcalino, al actuar sobre la portlandita, puede formar sulfato cálcico en la fase líquida de los poros de la pasta de cemento. Estos esquemas pueden explicar y justificar la presencia de sulfato cálcico (junto con sulfato alcalino) en los esquemas 14), 15), 15-I), 17), 17-I) y 18 y, en tal sentido, propiciar dicha presencia e, indirectamente, tales procesos. De cualquier modo se trataría de un problema complejo de solubilidades y concentraciones, en función de las condiciones en cada caso.

En consecuencia, como más verosímilmente representativos de los complicados procesos de un ataque al hormigón por sulfatos alcalinos. con formación de ettringita, de hidróxido alcalino, y circunstancialmente de gibbsita y de portlandita secundaria, se retienen los prosos 1), 6), 8), 12), 14) y 16 ).

Por implicar la participación de sulfato cálcico y ser el único que produce portlandita secundaria, y por no consumir portlandita primaria, el esquema 14) parece condicionado a los esquemas 1), 6), 8), previos o simultáneos, desplazados hacia la derecha, es decir, produciendo consumo de portlandita primaria, como ya se mencionó antes.

En definitiva, parece ser que en todo caso -y como señala S. CHATTERJI (7) citado por los autores (5)-, es indispensable la presencia y participación de hiddóxido cálcico - portlandita primaria - para que en un hormigón con áridos reactivos se produzca simultáneamente ettringita, hidróxido alcalino generador de la reacción árido-álcalis $\longrightarrow$ y por tanto de los geles isomorfos inherentes a la misma-, y (circunstancialmente) portlandita secundaria y gibbsita. En esto radica una de las varias razones ya expuestas para explicar el hecho, reconocido con toda generalidad (1) (2) (5) (7), de que los cementos puzolánicos contribuyen a inhibir la reacción árido-álcalis, eliminando o reduciendo los efectos perniciosos de la misma.

Otra razón aplicable a este caso, en que junto a la reacción árido-álcalis tiene lugar la formación de ettringita, es la influencia que las puzolanas de los cementos puzolánicos, y sobre todo las más ricas en sílice activa, precisamente, tienen en la formación, y más aún en la estabilidad, de dicho compuesto expansivo, en el sentido de que su hidrólisis aumenta al aumentar la relación puzolana/ettringita, más en agua destilada que en disolución saturada de sulfato cálcico, y más en ésta que en disolución saturada de hidróxido cálcico, hechos que son conocidos desde antiguo y han sido expuestos y confirmados en repetidas ocasiones.

En cualquier caso, y como indican los autores PETTIFER y NIXON (5), sería preciso profundizar en los estudios experimentales para confirmar o rechazar alguno de los esquemas expuestos -o establecer otros-, y más concretamente para precisar las condiciones en las que se puede formar portlandita secundaria -esquema 14) o análogos-, o gibbsita -esquema 16) o análogos-, en cada caso.

\section{R E F E R E N C I A S}

(1) Veronelli, D. J. E.: Comunicaciones privadas a J Calleja (1978 y 1979). 
(3) Calleja, J.: "La reacción árido-álcalis. Disquisıción aserca del texto de una conferencia dada sobre el tema por el Dr. Dante J. E. Veronelli", Cemento-Hormigón, 50 (542), 35-52 (Enero 1979).

(4) Chlieja, J.: "Durabilidad". Principal Report, 'Tema 7-II. 7." Congreso Internacional de Quimica del Cemento. Paris, Julio 1980.

(5) Pettifer, K. y Nixon, P. J.: "Alkali-metal sulphate - A factor common to both aggregate reaction and sulphate attack on concrete". Cement and Concrete Research, 10, 173-181 (1980).

(6) Diamond, S.: Citado en Refs. 4 y 5. Cement and Concrete Research, 5 (4), 329-346 (1975).

(7) Chatterui, S.: Citado en Refs. 4 y 5. Cement and Concrete Research, 9, 185-188 (1979). 